OPEN ACCESS

Edited by:

Francisco Ciruela,

Universitat de Barcelona, Spain

Reviewed by:

Christoph Anacker,

Columbia University, USA

Luis F. Callado,

University of the Basque Country,

Spain

${ }^{*}$ Correspondence:

Gregor Hasler

gregor.hasler@puk.unibe.ch,

g.hasler@bluewin.ch

Specialty section:

This article was submitted to

Neuropharmacology,

a section of the journal

Frontiers in Psychiatry

Received: 26 October 2015

Accepted: 15 December 2015

Published: 11 January 2016

Citation:

Slokar G and Hasler G (2016) Human

Endogenous Retroviruses as

Pathogenic Factors in the

Development of Schizophrenia.

Front. Psychiatry 6:183.

doi: 10.3389/fpsyt.2015.00183

\section{Human Endogenous Retroviruses as Pathogenic Factors in the Development of Schizophrenia}

\author{
Gorjan Slokar and Gregor Hasler* \\ Psychiatric University Hospital, University of Bern, Bern, Switzerland
}

Schizophrenia is a complex disorder, characterized by the interplay between genetic and environmental factors. Human endogenous retroviruses (HERVs), genetic elements that originated from infections by exogenous retroviruses millions of years ago, comprise $\sim 8 \%$ of the human genome. Here, we provide a comprehensive review of accumulating evidence, detailing HERV aberrancies associated with schizophrenia. Studies examining the genome, transcriptome, and proteome of individuals with schizophrenia provide data that support the association of these viral elements with the disorder. Molecular differences can be found within the central nervous system and peripheral tissues. However, additional studies are needed to substantiate the reported link and to address several discrepancies among previous investigations. We further discuss potentially relevant pathogenic mechanisms to the development of schizophrenia.

Keywords: schizophrenia, human endogenous retrovirus, HERV-W, pathogenesis, gene-environment interaction, infectious

\section{INTRODUCTION}

The pathogenesis of schizophrenia is presently hypothesized to involve complex interactions between genetic and environmental factors (1). Occurrence of such interactions during critical phases of human neurodevelopment is thought to underlie disease initiation (2). Recent efforts have identified numerous candidate gene loci that confer increased susceptibility to this devastating disorder (3-5). Considering environmental influences, infectious agents have long been suspected to be involved in the etiology of schizophrenia (6), and accumulating evidence supports this theory. Being born during winter and spring (7) and living in relatively more urbanized areas (8) are both linked to an increased risk of exposure to infectious pathogens and to the development of schizophrenia. Prenatal maternal infections by Toxoplasma gondii, influenzavirus, and HSV-2 heighten the child's risk of being diagnosed with the disorder at a later stage in life (9). Inflammatory markers associated with infection are elevated in peripheral blood (10) and cerebrospinal fluid (CSF) samples of subjects with schizophrenia (11), and occurrence of higher values of certain cytokines during childhood increases the probability of developing psychosis as a young adult (12). Moreover, several gene loci associated with schizophrenia are found in genomic regions that determine the response of the immune system to pathogens (4). However, the mechanism by which diverse infectious agents might be involved in the etiology of schizophrenia has not yet been identified. In this review, we summarize evidence regarding a possible missing link between infectious pathogens and schizophrenia: the so-called human endogenous retroviruses (HERVs).

Endogenous retroviruses (ERVs) are endogenous viral elements that are derived from past infections of germ cells by exogenous retroviruses. The first infection of a human ancestor by an 
exogenous retrovirus is estimated to have taken place around 100 million years ago (13). Many more integration events have occurred since, until possibly as recently as 100,000 years ago (14), which has resulted in HERV comprising about $8 \%$ of the present human genome, distributed over several hundred thousand loci (15). Given the time scale of these integrations, currently, these elements are shared among different human populations, with only a few loci displaying insertional polymorphisms (presence/ absence) $(16,17)$. Nearly all HERVs have accumulated mutations that prevent them from forming infectious virions or retrotransposing within the cell. Epigenetic silencing mechanisms further interfere with these processes, therefore, the inheritance of HERVs is limited to the classical Mendelian pattern. HERVs can be grouped into three classes, according to their similarities with existing exogenous retrovirus genera. Class I, Class II, and Class III contain Gammaretrovirus-like, Betaretrovirus-like, and Spumavirus-like HERVs. They can be further classified into "Families," based on varying criteria such as sequence similarity or primer-binding sites. HERV classification has been extensively reviewed (18-20). HERVs exhibit the same basic gene structure as do exogenous retroviruses: 5'-LTR-gag-pro-pol-env-LTR-3'. The long terminal repeats (LTRs) contain promoter and enhancer sequences, necessary for transcription. Gag, pro, pol, and env encode the different proteins needed for the retroviral life cycle. However, most HERVs simply consist of a solitary LTR, missing the retroviral genes and the second LTR. Such HERVs are believed to have originated from homologous recombination of $5^{\prime}$ and $3^{\prime}$ LTRs. Several physiological functions of HERVs have started to be recognized in recent years. Recombination events involving HERV s have contributed substantially to the current structure of the human genome (21). LTRs of retroviral elements have been incorporated into the regulatory region of human genes to serve as promoters or enhancers (22). Apart from their significance for protein-coding genes, the role of HERVs in the evolution and regulation of non-coding RNAs is increasingly being recognized (23). In addition to their importance at the DNA level, HERVs themselves are also transcribed into RNA, depending on tissue type and the developmental stage $(24,25)$. The physiological significance of most of these transcripts has not yet been clearly established. Some transcripts are translated into proteins and perform essential functions in the host. The most well-studied instance is the function of $e n v$-derived proteins in the formation of the syncytiotrophoblast (26). HERV protein expression can also be observed in other tissues of healthy human individuals; however, the physiological role of these proteins has not yet been elucidated (27-29).

\section{HERVs AND SCHIZOPHRENIA}

Human endogenous retrovirus have been implicated in various diseases, such as cancer (30), autoimmune diseases (31-33), and neuropsychiatric illnesses. In the following summary, we will focus on HERV-related findings in schizophrenia. Our summary is structured based on the molecular focus of each study (genetics, RNA expression, protein expression, and antibodies), and the different tissues investigated (brain, CSF, leukocytes, or plasma/ serum).

\section{DNA Studies}

DNA studies focusing on the role of HERVs in schizophrenia have tried to determine whether subjects with schizophrenia possess unknown HERV insertions/deletions or known polymorphisms correlate with the disease; an overview of the included studies has been presented in Table 1. In a study of three monozygotic twin pairs discordant for schizophrenia, a sequence designated schizophrenia-associated retrovirus

\begin{tabular}{|c|c|c|c|}
\hline Study & Subjects & Target sequence & Main findings \\
\hline Deb-Rinker et al. (34) & $\begin{array}{l}3 \text { Monozygotic twin pairs discordant } \\
\text { for schizophrenia }\end{array}$ & Retroviral pol & SZRV-1a identified by representational difference analysis \\
\hline Yolken et al. (37) & $\begin{array}{l}\text { Schizophrenia } \\
\text { Healthy controls }\end{array}$ & c-myc pseudogene & Pseudogene present in some affected subjects \\
\hline Deb-Rinker et al. (35) & $\begin{array}{l}8 \text { Schizophrenia } \\
21 \text { Healthy controls }\end{array}$ & SZRV-2 & No differences detected \\
\hline Otowa et al. (38) & $\begin{array}{l}17 \text { Schizophrenia } \\
181 \text { Healthy controls }\end{array}$ & HERV-K115 & $\begin{array}{l}\text { HERV-K115 presence not increased. Tendency toward increased } \\
\text { presence in subjects with younger onset }(p=0.057)\end{array}$ \\
\hline Dickerson et al. (39) & $\begin{array}{l}229 \text { Schizophrenia } \\
136 \text { Healthy controls }\end{array}$ & HERV-K18 SNPs ${ }^{b}$ & $\begin{array}{l}\text { Haplotype associated with type } 2 \text { diabetes in SZ ( } p<0.001 \text {, odds } \\
\text { ratio }=9)\end{array}$ \\
\hline Nyegaard et al. (40) & $\begin{array}{l}750 \text { Schizophrenia } \\
1214 \text { Healthy controls }\end{array}$ & HERV-K18 SNPs & $\begin{array}{l}\text { No association with type } 2 \text { diabetes. Tendency toward association } \\
\text { with type } 2 \text { diabetes in younger schizophrenia subjects ( } p=0.18)\end{array}$ \\
\hline Perron et al. (36) & $\begin{array}{l}59 \text { Schizophrenia } \\
110 \text { Bipolar disorder } \\
105 \text { Healthy controls }\end{array}$ & HERV-W env (MSRV') & $\begin{array}{l}\text { Copy number of HERV-W env reduced in schizophrenia } \\
(p<0.001) \text { and bipolar disorder }(p=0.001)\end{array}$ \\
\hline
\end{tabular}

Here, we present a summary of genetic studies, examining genetic differences directly or indirectly relating to HERVs in individuals with schizophrenia and healthy controls. "Target sequence" denotes the sequences of the human genome used for PCR.

aschizophrenia-associated retrovirus 1.

' Single nucleotide polymorphisms.

cMultiple sclerosis-associated retrovirus. 
1 (SZRV-1) was amplified by polymerase chain reaction (PCR). Subsequent application of representational difference analysis yielded increased quantities of this sequence in all three affected twins (34). The observed sequence exhibited high similarity to the multiple sclerosis-associated retrovirus (MSRV). Three other sequences were further amplified, which were not increased in all the affected twins. When one of these sequences, termed SZRV-2, was used as a probe for Southern blot, the detection rate did not differ between eight subjects and their matched controls (35). Investigation of HERV-W env (MSRV subtype) copy number by real-time polymerase chain reaction (qPCR) in 59 subjects with schizophrenia revealed a significantly reduced copy number in the subjects compared to healthy controls (36).

A study investigating HERV-K115, a member of the HERVK(HML-2) family displaying insertional polymorphism, found no association between HERV-K115 and schizophrenia (38). However, subjects with younger-onset schizophrenia exhibited a higher frequency of this retroviral element (38). On examining single nucleotide polymorphisms (SNPs) within the HERV-K18 locus in 229 schizophrenia subjects, one study found that a haplotype of two SNPs increased the risk of developing type 2 diabetes (odds ratio =9) (39). A weaker association with type 2 diabetes was also found for several other SNPs of this locus (39). Another study could not confirm this association in a sample of 750 schizophrenia subjects, but observed a tendency toward an increased type 2 diabetes risk in subjects with younger-onset schizophrenia (40). They also found no association between any of the measured SNPs and an increased risk of developing schizophrenia.

Examination of the DNA of individuals with schizophrenia for the presence of processed pseudogenes of the c-myc transcription factor, which have not been detected in the genome of healthy individuals, revealed the presence of pseudogenes in some individuals with schizophrenia, while none were observed in healthy controls (37). Processed pseudogenes arise from the reverse transcription and integration of cellular messenger RNA into the nuclear DNA by retrotransposons.

\section{RNA Studies}

An overview of the RNA studies has been presented in Table 2. In a study of brain tissue obtained from four schizophrenia subjects, application of primers specific for the retroviral pol region revealed that $45 \%$ of transcripts in schizophrenia subjects were homologous to HERV-W (MSRV) compared to $10 \%$ in controls (37). A subsequent study found the level of HERV-W transcripts to be significantly increased and that of ERV9 transcripts to be significantly decreased in the frontal cortex of five schizophrenia subjects (41). In a microarray study involving 35 schizophrenia subjects and 35 bipolar disorder subjects, no differences were found in the levels of HERV-W or ERV9 transcripts between subjects and controls; however, HERV-K10 transcripts were significantly increased in individuals with schizophrenia and bipolar disorder (42). Reverse transcription-polymerase chain reaction (RT-PCR) was also applied, targeting different HERV env sequences, and found no differences between samples obtained from healthy controls and individuals with schizophrenia (42). To evaluate the effect of psychiatric medication on HERV transcription, one study measured
HERV RNA in different cell lines and in 18 brain samples obtained from subjects with schizophrenia and 20 brain samples obtained from subjects with bipolar disorder. Valproic acid (VPA) was found to induce increased RNA expression of certain HERVs in vitro with a particularly strong effect on the RNA expression of HERV-W and ERV9, but not on HERV-K (43). Tested antipsychotics had a minimal impact on HERV transcription (43). In the same study, ERV9 transcripts were significantly elevated in schizophrenia, and ERV9 and HERV-K (HML-2) transcripts were significantly elevated in bipolar disorder, compared to that observed in healthy controls independent of VPA treatment. HERV-W expression was not associated with schizophrenia or bipolar disorder. However, VPA-treated schizophrenia subjects exhibited significantly elevated HERV-W RNA levels, compared to those observed in untreated schizophrenia subjects. No such association could be found in VPA-treated bipolar disorder subjects.

In a study of CSF samples obtained from 35 subjects with recent-onset schizophrenia and 20 subjects with chronic schizophrenia, HERV RNA was detected in 10/35 subjects of the former group, $1 / 20$ subjects of the latter group, and in none of the subjects of the non-inflammatory neurologic disease and healthy control group. The sequences identified belonged to the HERV-W, ERV9, and HERV-FRD families, with HERV-W constituting the majority of the sequences, and were reported to be particle-associated (41). Illness duration and symptom severity were not associated with the increase in HERV transcripts.

Based on their findings in the CSF, the presence of retroviral gag sequences was examined in the plasma samples of 54 recentonset schizophrenia subjects. Particle-associated HERV-W RNA was found in 9/54 subjects and 2/46 controls (44). Five of the nine subjects whose plasma tested positive for particle-associated HERV-W RNA were also previously found to harbor retroviral RNA in their CSF, while only three subjects whose CSF tested negative for particle-associated HERV-W RNA were found to harbor retroviral RNA in their plasma. HERV-W-positive subjects showed a tendency toward increased acuity of psychotic symptoms $(p=0.06)$. Medication intake was not associated with HERV status. Another study detected HERV-W env RNA in the plasma of 42/118 recent-onset schizophrenia subjects, and in none of the controls (47).

ERV9 RNA was detected in the leukocytes of 20/58 subjects with recent-onset schizophrenia and 0/38 healthy controls (45). Another study tested peripheral blood monocular cells (PBMCs) of 30 first-episode schizophrenia subjects for the presence of HERV-W env and gag sequences. Multiple HERV-W elements were transcribed in both groups. gag transcripts were increased twofold in subjects with schizophrenia compared to those in healthy controls (46). A HERV-W element on 11q13.5 was identified as the main contributor. This element is inserted into the second intron of the putative gene PTD015. No differences were seen in env transcripts. gag transcripts exhibited a significant inverse relationship with illness duration. Symptom severity and medication intake was not associated with increased gag transcripts. The state of activation of PBMCs was further assessed by measuring the transcripts of two genes involved in metabolic pathways; however, no differences were found between schizophrenia subjects and healthy controls. Moreover, lymphocyte culture stimulation 
TABLE 2 | Transcriptome studies on HERVs in schizophrenia.

\begin{tabular}{|c|c|c|c|c|}
\hline Study & Subject(s) & Specimen & Target sequence & Main findings \\
\hline \multicolumn{5}{|c|}{ Brain (postmortem) } \\
\hline Yolken et al. (37) & $\begin{array}{l}4 \text { Schizophrenia } \\
4 \text { Bipolar disorder } \\
6 \text { Healthy controls }\end{array}$ & Frontal cortex & Retroviral pol & $\begin{array}{l}\text { Increased HERV-W (MSRV) in schizophrenia. Increased } \\
\text { HERV-K10 in bipolar disorder }\end{array}$ \\
\hline Karlsson et al. (41) & $\begin{array}{l}5 \text { Schizophrenia } \\
6 \text { Healthy controls }\end{array}$ & Frontal cortex & Retroviral pol & $\begin{array}{l}\text { Increased HERV-W }(p<0.0001) \text {. Significantly decreased } \\
\text { ERV9 }(p<0.0001)\end{array}$ \\
\hline \multirow[t]{2}{*}{ Frank et al. (42) } & $\begin{array}{l}35 \text { Schizophrenia } \\
35 \text { Bipolar disorder } \\
35 \text { Healthy controls }\end{array}$ & $\begin{array}{l}\text { Prefrontal } \\
\text { cortex }\end{array}$ & Retroviral pol & $\begin{array}{l}\text { Increased HERV-K10 in schizophrenia }(p<0.05) \text { and } \\
\text { bipolar disorder }(p<0.01)\end{array}$ \\
\hline & $\begin{array}{l}7 \text { Schizophrenia } \\
7 \text { Healthy controls }\end{array}$ & & $\begin{array}{l}\text { HERV-W env, } \\
\text { HERV-FRD env, } \\
\text { HERV-K env }\end{array}$ & No significant differences \\
\hline Diem et al. (43) & $\begin{array}{l}18 \text { Schizophrenia } \\
20 \text { Bipolar disorder } \\
18 \text { Healthy controls }\end{array}$ & $\begin{array}{l}\text { Prefrontal } \\
\text { cortex }\end{array}$ & $\begin{array}{l}\text { HERV-W pol, } \\
\text { ERV9 pol, HERV- } \\
\text { K(HML-2) gag }\end{array}$ & $\begin{array}{l}\text { ERV9 increase in schizophrenia }(p<0.01) \text {. ERV9 and } \\
\text { HERV-K(HML-2) increases in bipolar disorder }(p<0.01) \\
\text { Increases independent of medication }\end{array}$ \\
\hline \multicolumn{5}{|l|}{ CSF } \\
\hline Karlsson et al. (41) & $\begin{array}{l}35 \text { Recent-onset schizophrenia or } \\
\text { recent-onset schizoaffective disorder } \\
20 \text { Chronic schizophrenia or chronic } \\
\text { schizoaffective disorder } \\
22 \text { Non-inflammatory neurologic disease } \\
30 \text { Healthy controls }\end{array}$ & $\begin{array}{l}\text { Cell-free } \\
\text { supernatant }\end{array}$ & Retroviral pol & $\begin{array}{l}\text { Increased HERV-W, ERV9 and HERV-FRD expression } \\
(p<0.001) 10 / 35,1 / 20,0 / 22,0 / 30\end{array}$ \\
\hline \multicolumn{5}{|l|}{ Blood } \\
\hline Karlsson et al. (44) & $\begin{array}{l}54 \text { Recent-onset schizophrenia or recent- } \\
\text { onset schizoaffective disorder or recent- } \\
\text { onset schizophreniform disorder } \\
46 \text { Healthy controls }\end{array}$ & Plasma & Retroviral gag & Increased HERV-W $(p=0.05)$ 9/54, 0/30 \\
\hline Huang et al. (45) & $\begin{array}{l}58 \text { Recent-onset schizophrenia } \\
38 \text { Healthy controls }\end{array}$ & Leukocytes & Retroviral pol & Increased ERV9 $(p<0.01)$ 20/58, 0/38 \\
\hline Yao et al. (46) & $\begin{array}{l}30 \text { Recent-onset schizophrenia } \\
26 \text { Healthy controls }\end{array}$ & $\mathrm{PBMCs}^{\mathrm{a}}$ & HERV-W env, gag & $\begin{array}{l}2.1 \text {-fold increase in gag, mainly from the element on } \\
11 q 13.5(p<0.01) \text {. No env increase }\end{array}$ \\
\hline Huang et al. (47) & $\begin{array}{l}118 \text { Recent-onset schizophrenia } \\
106 \text { Healthy controls }\end{array}$ & Plasma & HERV-W env & Increased HERV-W env $(p<0.01)$ 42/118, 0/106 \\
\hline Perron et al. (36) & $\begin{array}{l}45 \text { Schizophrenia } \\
91 \text { Bipolar disorder } \\
73 \text { Healthy controls }\end{array}$ & PBMCs & HERV-W env & $\begin{array}{l}\text { Increased transcripts in schizophrenia }(p=0.01) \text { and } \\
\text { bipolar disorder }(p<0.0001) \text {. Association remained after } \\
\text { exclusion of VPA-treated subjects }\end{array}$ \\
\hline
\end{tabular}

Here, we present a summary of studies examining differences in HERV transcription in individuals with schizophrenia in comparison to healthy controls. The table is divided into studies examining brain, CSF, and blood samples. "Specimen" denotes the exact origin/processing of samples. "Target sequence" denotes the sequence of the retroviral genome that was used for PCR.

aperipheral blood monocular cells.

from three blood donors did not affect transcripts of the HERV-W gag gene detected in schizophrenia subjects. Exploration of the presence of HERV-W (MSRV) env sequences in PBMCs of 45 individuals with chronic schizophrenia revealed a significant increase in transcripts compared to that observed in controls and a significant decrease compared to that observed in bipolar disorder subjects (36). The association remained after exclusion of VPA-treated subjects. No association of HERV transcripts was found with illness duration or symptom severity. However, seropositivity against $T$. gondii was significantly increased in HERV-W-positive bipolar disorder and schizophrenia subjects, merged as a single group. Seropositivity against Herpes simplex virus $1 / 2$ and Cytomegalovirus did not correlate with HERV-W detection.

\section{Protein Studies}

An overview of the protein studies has been presented in Table 3. Reverse transcriptase activity was measured in the cerebellum of 12 schizophrenia subjects by the product-enhanced reverse transcriptase assay and found to be significantly increased compared to that in the controls (37). Significantly decreased levels of HERV-W GAG proteins were detected in the anterior cingulate gyrus and the hippocampus of 15 schizophrenia, 15 bipolar disorder, and 15 major depressive disorder subjects by immunohistochemistry (28). GAG proteins were expressed in neurons and astroglial cells. Analysis of GAG by western blotting yielded multiple bands, leading the researchers to conclude that multiple HERV-W elements contribute to this expression. The number of immune cells in brain tissue did not differ between subjects and controls. No association could be found between HERV-W GAG expression and serological status for T. gondii, Herpes simplex virus $1 / 2$, and Cytomegalovirus.

In serum samples, HERV-W GAG proteins were found in 24/49 individuals with schizophrenia and 2/49 controls, and HERV-W ENV in 23/49 individuals with schizophrenia and $1 / 30$ controls (49). Detection of both proteins significantly 
TABLE 3 | HERV protein studies in schizophrenia.

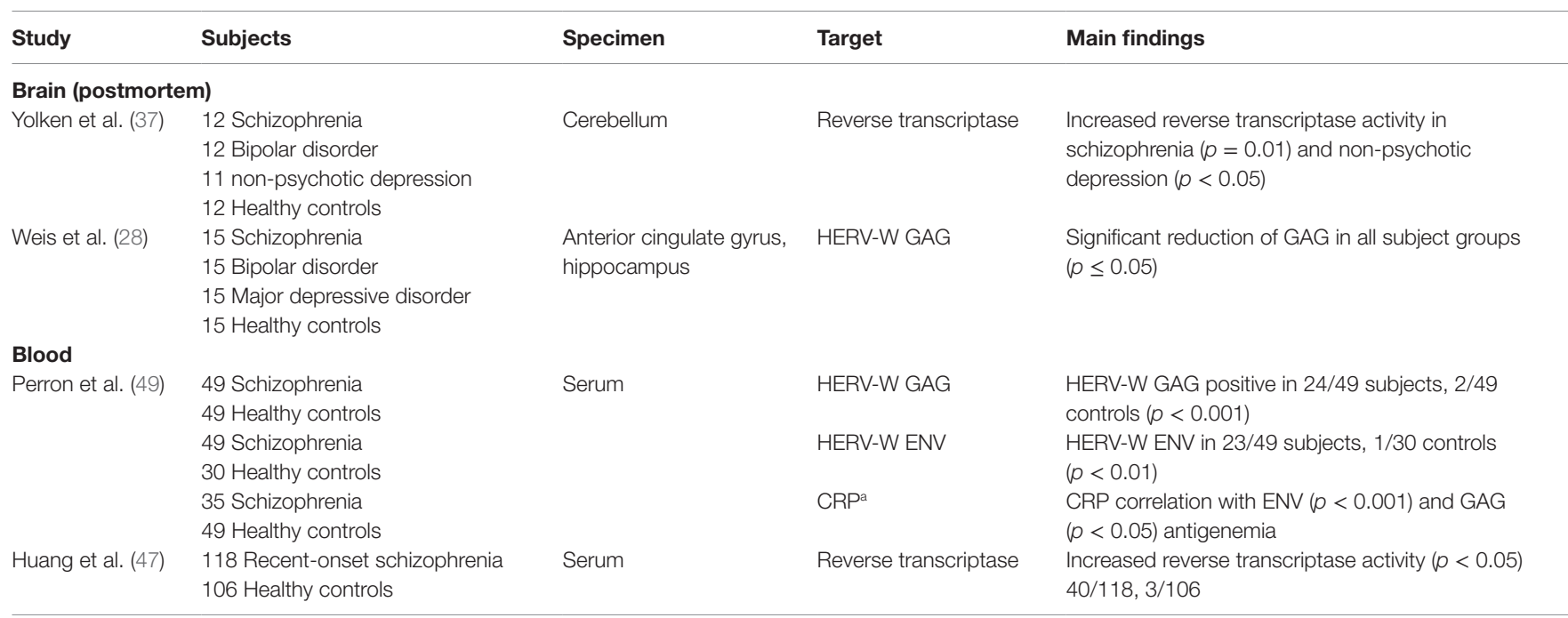

Here, we present a summary of studies examining differences in protein expression relating to HERVs in schizophrenia subjects in comparison to healthy controls. The table is divided into studies examining brain and blood samples. "Specimen" denotes the exact origin/processing of the samples. "Target" denotes the retroviral protein assayed in the study. ${ }^{a}$ C-reactive protein

correlated with C-reactive protein (CRP) levels. Medication intake was not associated with seropositivity for HERV-W proteins. Measurement of reverse transcriptase activity in the serum of recent-onset schizophrenia subjects by quantitative reverse transcription PCR (RT-qPCR) yielded activity of this enzyme in 40/118 schizophrenia subjects and 3/106 controls (47).

\section{Antibody Studies}

Antibodies directed against a particular retroviral antigen can bind to homologous proteins of related retroviruses. Protein antigens of readily available retroviruses can, therefore, be used to detect antibodies directed against unidentified retroviruses. An overview of antibody studies has been presented in Table 4. HIV-1 western blot assay was applied to screen 38 schizophrenia subjects for the presence of retroviral antibodies. Antibodies were present in 21/38 subjects and 4/16 controls (50). Application of protein antigens of the betaretroviruses Mason-Pfizer monkey virus (MPMV), mouse mammary tumor virus (MMTV), simian retrovirus 5 (SRV-5), and Gammaretrovirus baboon endogenous virus $(\mathrm{BaEV})$ revealed a significantly increased incidence of antibodies against MPMV, BaEV, and SRV-5 in 17 first-episode schizophrenia subjects (51). The largest differences were seen in antibodies against MPMV. The authors, therefore, assessed antibody levels against this species by immunoassay and immunoblot in 38 first-episode schizophrenia subjects and found that $11 / 38$ subjects and $1 / 27$ controls had antibodies above the set threshold (51). Follow-up of four seropositive subjects after 1 year revealed that two had reverted to a seronegative status and optical densities were diminished in the other two subjects. Using MPMV, the Gammaretrovirus murine leukemia virus (MuLV) and the Lentivirus feline immunodeficiency virus (FIV), significantly increased antibody levels against MPMV and MuLV were detected in 163 subjects with recent-onset psychosis, but not in 268 multi-episode schizophrenia subjects (52). These were directed against gag, pol, and env encoded proteins. Since the authors could not find any nucleic acids of the tested retroviruses in the subject sera and multiple regions of homology for both retroviruses were found within the human genome, they concluded that the detected antibodies likely arose from activation of homologous HERVs. Increased antibodies were not associated with symptom severity, illness duration, or medication intake. Antibodies against the ERV9 family were measured by in vitro expression of ERV9 POL proteins. Antibodies were present in the sera of some subjects, who also tested positive for ERV9 RNA, and absent in the sera of controls (45).

\section{Potential Contributions of HERVs to the Pathogenesis of Schizophrenia}

Different downstream effects of increased HERV activation may be involved in the pathogenesis of schizophrenia. Proinflammatory properties of HERV DNA, RNA, or proteins may be at the root of the immune aberrancies observed in individuals with schizophrenia. Normally, the immune response against viruses involves the detection of viral DNA and RNA within the cell. It has been proposed that HERV DNA and/or RNA might also activate such pathways (53). Such a mechanism is supported by a recent study, which details the exploitation of endogenous retroviral transcripts by the immune system, to induce a humoral immune response without the help of T-cells (54). More definite evidence has been obtained regarding the pro-inflammatory effects of HERV proteins. HERV-W ENV has been shown to induce an innate immune response via interaction with Toll-like receptor 4 (TLR4) and promote a TH1-like response, stimulating the release of IL- 6 , TNF- $\alpha$, and IL-1 $\beta$ (55). The same protein also generates cytokine production in astroglial cells, induces death of oligodendrocytes, and may evoke superantigen-like effects $(56,57)$. In vivo, ENV and GAG antigenemia within serum 
TABLE 4 | Retroviral antibodies in schizophrenia.

\begin{tabular}{|c|c|c|c|}
\hline Study & Subjects & Target & Main findings \\
\hline Hart et al. (50) & $\begin{array}{l}38 \text { Schizophrenia } \\
16 \text { Healthy controls }\end{array}$ & HIV-1 & Increased antibodies to HIV-1 antigens in SZ $(p=0.05) 21 / 38,4 / 16$ \\
\hline Lillehoj et al. (51) & $\begin{array}{l}15 \text { Recent-onset schizophrenia } \\
27 \text { Healthy controls } \\
38 \text { Recent-onset schizophrenia } \\
27 \text { Healthy controls }\end{array}$ & $\begin{array}{l}\text { MPMV }{ }^{a} \text { GAG, BaEV }{ }^{\circ} \text { ENV/GAG, } \\
\text { MMTV }^{c} \text { GAG, SRV-5 }{ }^{d} \text { GAG } \\
\text { MPMV GAG }\end{array}$ & $\begin{array}{l}\text { Increased incidence of antibodies to MPMV }(p<0.001) \text {, BaEV } \\
(p<0.05) \text {, and SRV-5 }(p<0.05) \text { antigens } \\
\text { Increased antibody levels against MPMV }(p<0.01) 11 / 38,1 / 27\end{array}$ \\
\hline Huang et al. (45) & $\begin{array}{l}58 \text { Recent-onset schizophrenia } \\
38 \text { Healthy controls }\end{array}$ & ERV9 POL & Antibodies present in some ERV9 RNA-positive subjects \\
\hline Dickerson et al. (52) & $\begin{array}{l}163 \text { Recent-onset psychosis } \\
268 \text { Multiple-episode schizophrenia } \\
235 \text { Healthy controls }\end{array}$ & MuLVe, MPMV, FIV & $\begin{array}{l}\text { Increased antibodies to MuLV and MPMV in recent-onset psychosis } \\
(p<0.001) \text {. No increase in multiple-episode schizophrenia }\end{array}$ \\
\hline
\end{tabular}

Here, we present a summary of studies examining the presence of retroviral antibodies in schizophrenia subjects and healthy controls. "Target" denotes the retroviruses/retroviral proteins used to assay the presence of antibodies.

aMason-Pfizer monkey virus.

${ }^{\circ}$ Baboon endogenous virus.

"Mouse mammary tumor virus.

${ }^{\circ}$ Simian retrovirus 5 .

eMurine leukemia virus.

${ }^{f}$ Feline immunodeficiency virus.

significantly correlate with CRP levels (49). Multiple studies have repeatedly linked an increase in CRP, IL- 6 , TNF- $\alpha$, and IL- $1 \beta$ to schizophrenia $(10,58)$, supporting inflammatory processes such as HERV-W-related ones being involved in the pathogenesis of this severe disorder.

Apart from its inflammatory effects, several other properties of HERV-W ENV have been proposed to be involved in the pathogenesis of schizophrenia. The human sodium-dependent neutral amino acid transporter type 1 (hASCT1) and hASCT2 have been established as the cellular receptors of HERV-W ENV (59). Both receptors play a role in the regulation of glutamatergic transmission in the brain (48), which has been found dysfunctional in schizophrenia. It has, therefore, been speculated that HERV-W ENV might contribute to schizophrenia by disturbing these pathways (44). Cell cultures infected by exogenous retroviruses, using an allelic receptor, do indeed exhibit impaired transport of the amino acids glutamine and alanine (60). Furthermore, a significant decrease in immunoreactivity of hASCT1 has been observed in neurons and astroglial cells of the cingulate cortex and hippocampus in individuals with schizophrenia, possibly reflecting occupation of the receptor by HERV-W ENV or receptor downregulation (48). Expression of HERV-W env in human glioma cells results in a two- to fourfold increase in expression of brain-derived neurotrophic factor (BDNF), neurotrophic tyrosine kinase receptor type 2 (NTRK2), and dopamine receptor D3 (DRD3) (47). BDNF aberrancies have been repeatedly associated with schizophrenia (61). In addition, the small conductance $\mathrm{Ca}^{2+}$-activated $\mathrm{K}^{+}$channel protein 3 (SK3), which has been linked to schizophrenia as well, is also increased after expression of HERV-W env within neuroblastoma cells (62). Rats, receiving bilateral injections of recombinant HERV-W ENV protein into the ventral region of the hippocampus, exhibit several neurological and behavioral abnormalities associated with schizophrenia, including increased sensitivity to the psychostimulant MK-801, a common feature of animal models of schizophrenia, and a strong emergence of stereotypic behaviors, which are associated with some schizophrenia subtypes (63).

Aside from the effects of HERV products, HERVs might also contribute to schizophrenia via their regulatory effects on cellular genes. One HERV-W element was identified within the regulatory region of the GABA receptor $\mathrm{B} 1$ gene $G A B B R 1$, which is down-regulated in schizophrenia (64). A HERV-K(HML-2) element was confirmed to act as an enhancer for the schizophreniaassociated gene $P R O D H$ (65). It has been hypothesized that increased activity of one HERV element may lead to widespread epigenetic silencing of the whole respective HERV family, resulting in aberrant quantities of cellular proteins whose transcription is controlled by the individual HERV elements, such as GABA receptor $\mathrm{B} 1$.

In addition to their significance for protein-coding genes, the role of HERVs in the regulation of non-coding RNA potentially constitutes another pathogenic mechanism. HERVs are associated with a large number of long non-coding RNAs (lncRNAs), where they mainly overlap with transcriptional start sites (66). LncRNAs have a broad spectrum of biological activities, e.g., they can affect transcription, chromatin state, or splicing patterns, and are thought to be of particular importance in brain development (67). Evidence is emerging for an involvement of these molecules in disorders of the brain, including schizophrenia (68-70). It remains to be demonstrated whether any of the HERV transcripts observed in individuals with schizophrenia constitute functional non-coding RNA.

\section{DISCUSSION}

The most consistent evidence of an association between HERVs and schizophrenia comes from studies on HERV transcripts in blood samples of individuals with schizophrenia. Four studies found an increase in HERV-W transcripts, one study found an increase in ERV9 transcripts. Studies in postmortem brain tissue 
and CSF of schizophrenia subjects partly confirm these findings in blood. One study in CSF found increased HERV-W transcripts. Three studies in brain tissue found increased HERV-W, increased HERV-K10, and increased ERV9 transcripts. One possible reason for discrepancies regarding the associated HERV families in all of these studies may be related to technical differences and the high copy number of HERVs within the human genome. The HERV-W family is comprised of an estimated 250 elements and nearly all of these contain mutations, such as stop-codons, frame-shifts, or deletions (71). This sequence heterogeneity makes it very difficult to design primers for PCR, to amplify all members of a particular HERV family (24). Owing to the use of distinct primers in each study, different subsets of HERVs were likely amplified.

Four studies targeting retroviral proteins confirm findings of differential HERV activity at the protein level. Two studies found an increased reverse transcriptase activity, which is unspecific to any particular HERV family. One study found decreased HERV-W GAG, while another study found increased HERV-W GAG and ENV. Causes for decreased HERV-W GAG protein levels in the brain of individuals with schizophrenia are unclear, given the increases of this protein in the blood and at the RNA level. The authors themselves speculate that interfering mechanisms by non-coding HERV transcripts, disrupting the translation of coding transcripts, may yield an explanation (28).

Findings of HERV RNA and proteins in individuals with schizophrenia are further corroborated by the consistent detection of retroviral antibodies in this population. Four studies detected increased antibody levels to a Lentivirus, betaretroviruses, gammaretroviruses, and to the Gammaretrovirus-like ERV9. Considering evidence detailing HERV abnormalities associated with schizophrenia and the lack of evidence of an involvement of exogenous retroviruses in schizophrenia so far, it appears very likely that these antibodies are directed against HERVs. These antibody studies may additionally provide evidence for an involvement of the Betaretrovirus-like HERV-K in schizophrenia, since only one RNA study found an association with HERV-K. However, the detected antibodies against betaretroviruses may also simply be a result of cross-reactivity between antibodies against the Gammaretrovirus-like HERV-W or ERV9.

There is some evidence, indicating copy number variations of HERV-W in schizophrenia. One study using representational difference analysis found an increase in copy number, while another using qPCR found a decrease. Both studies suggest genomic alterations in schizophrenia subjects; however, more conclusive research is needed in this area.

There is preliminary evidence for the existence of c-myc pseudogenes in schizophrenia subjects. Although these have only been found in one study, they are of potential interest because this gene is mainly expressed during fetal development and may, thus, provide insights into the temporal development of environmental and genetic contributions to schizophrenia (37). These findings may, however, not only point to an involvement of HERVs but also point to the action of other retrotransposons, such as long interspersed nuclear elements (LINEs), also shown to be increasingly active in schizophrenia $(37,72)$.

Association of increased HERV activity with illness duration of schizophrenia subjects is debatable. Based on the initial findings in CSF, which were limited to recent-onset schizophrenia subjects, subsequent studies focused on subjects in the early symptomatic disease stages. Retroviral antibodies also seem to be exclusively found at disease onset. However, two studies analyzing the blood of chronic schizophrenia subjects and four post-mortem brain studies detected HERV abberrancies in later disease stages. Three studies found no association between illness duration and HERV expression/antibodies, while one study found an inverse relationship between illness duration and HERV RNA levels.

One in vitro study reported the influence of VPA on the transcription of HERV-W and ERV9. Two of six in vivo studies found an association between VPA intake and HERV-W expression, while the other four did not report any association. However, only one of these four studies separately analyzed different psychiatric drugs. The association of HERV-W with schizophrenia remained in one of the two positive studies after exclusion of subjects treated with VPA. It appears that VPA contributes, in part, to the HERV-related phenomena observed in schizophrenia. Future research will have to assess previous and current VPA intake to eliminate this confounding variable.

In vitro experiments have demonstrated that HERV expression can be induced by activation of monocytes (73). It has, therefore, been hypothesized that HERV-related findings in schizophrenia might simply be the result of increased immune cell activation or infiltration (42). However, the results of two studies in schizophrenia subjects do not support this premise, since immune cell activation in the blood and immune cell number in the brain did not differ in individuals with schizophrenia. Based on research detailing activation of HERVs after infection of immune (74) and neural cells (75-77), it has been speculated that transcriptional deregulation of HERVs in schizophrenia might be the result of an initial infectious event. HERV activation has been demonstrated in infections by schizophrenia-associated pathogens HSV-1 (76), T. gondii (75), and influenzavirus $(76,77)$. One study in schizophrenia subjects partially corroborates this hypothesis, since antibodies against $T$. gondii were associated with increased HERV-W transcripts, although no association was found with herpes viruses. Another smaller study in schizophrenia subjects could not find any association between infectious agents and HERV-W expression. It is important to note that HERV expression modulation in infection models is not limited to a single HERV family, such as HERV-W, but involves many different HERV families. It remains to be demonstrated whether the action of certain infectious agents may ultimately lead to similar changes in HERV expression over time, as have been observed in schizophrenia.

The association of HERV-W and schizophrenia is not highly specific. Bipolar disorder has also been associated with this HERV family, as has been reported in this review. Schizophrenia and bipolar disorder share approximately $60 \%$ of genetic risk factors (78) and exhibit similar brain structural abnormalities, such as reductions in total brain gray and white matter, and increased lateral ventricular volume (79). HERV-W has also been repeatedly linked with multiple sclerosis $(80,81)$. Schizophrenia and multiple sclerosis have been reported to share certain epidemiologic features, such as age of onset, season of birth, and geographic 
distribution (41). However, schizophrenia, bipolar disorder, and multiple sclerosis also exhibit differences regarding the molecular characteristics of HERV-W, which may be in part responsible for the observed differences in phenotype. As previously reported in one study, the DNA copy number of HERV-W is reduced in schizophrenia and bipolar disorder subjects. Conversely, examination of DNA copy number of HERV-W in multiple sclerosis subjects revealed increased copy numbers of this HERV family in the diseased subjects compared to healthy controls (82). Furthermore, the nucleotide sequences of examined HERV-W DNA and RNA slightly differ among all three disorders (36), possibly reflecting different subsets of HERV-W elements being activated.

Taken together, preliminary evidence suggests that differential activity of some HERV families is associated with schizophrenia in at least a subgroup of individuals with schizophrenia. The most frequently associated family is Gammaretrovirus-like HERV-W and to a lesser extent Gammaretrovirus-like ERV9 and Betaretrovirus-like HERV-K (HML-2). Limitations of the current evidence include the small number of studies, the high

\section{REFERENCES}

1. van Os J, Rutten BP, Poulton R. Gene-environment interactions in schizophrenia: review of epidemiological findings and future directions. Schizophr Bull (2008) 34:1066-82. doi:10.1093/schbul/sbn117

2. Rapoport JL, Giedd JN, Gogtay N. Neurodevelopmental model of schizophrenia: update 2012. Mol Psychiatry (2012) 17:1228-38. doi:10.1038/mp.2012.23

3. Van Winkel R, Esquivel G, Kenis G, Wichers M, Collip D, Peerbooms $\mathrm{O}$, et al. REVIEW: genome-wide findings in schizophrenia and the role of gene-environment interplay. CNS Neurosci Ther (2010) 16:e185-92. doi:10.1111/j.1755-5949.2010.00155.x

4. Corvin A, Morris DW. Genome-wide association studies: findings at the major histocompatibility complex locus in psychosis. Biol Psychiatry (2014) 75:276-83. doi:10.1016/j.biopsych.2013.09.018

5. Schizophrenia Working Group of the Psychiatric Genomics Consortium. Biological insights from 108 schizophrenia-associated genetic loci. Nature (2014) 511:421-7. doi:10.1038/nature13595

6. Yolken RH, Torrey EF. Are some cases of psychosis caused by microbial agents? A review of the evidence. Mol Psychiatry (2008) 13:470-9. doi:10.1038/ $\mathrm{mp} .2008 .5$

7. Davies G, Welham J, Chant D, Torrey EF, McGrath J. A systematic review and meta-analysis of Northern Hemisphere season of birth studies in schizophrenia. Schizophr Bull (2003) 29:587-93. doi:10.1093/oxfordjournals.schbul. a007030

8. Vassos E, Pedersen CB, Murray RM, Collier DA, Lewis CM. Meta-analysis of the association of urbanicity with schizophrenia. Schizophr Bull (2012) 38:1118-23. doi:10.1093/schbul/sbs096

9. Brown AS, Derkits EJ. Prenatal infection and schizophrenia: a review of epidemiologic and translational studies. Am J Psychiatry (2010) 167:261-80. doi:10.1176/appi.ajp.2009.09030361

10. Upthegrove R, Manzanares-Teson N, Barnes NM. Cytokine function in medication-naive first episode psychosis: a systematic review and meta-analysis. Schizophr Res (2014) 155:101-8. doi:10.1016/j.schres.2014.03.005

11. Hayes LN, Severance EG, Leek JT, Gressitt KL, Rohleder C, Coughlin JM, et al. Inflammatory molecular signature associated with infectious agents in psychosis. Schizophr Bull (2014) 40:963-72. doi:10.1093/schbul/sbu052

12. Khandaker GM, Pearson RM, Zammit S, Lewis G, Jones PB. Association of serum interleukin 6 and C-reactive protein in childhood with depression and psychosis in young adult life: a population-based longitudinal study. JAMA Psychiatry (2014) 71:1121-8. doi:10.1001/jamapsychiatry.2014.1332

13. Lee A, Nolan A, Watson J, Tristem M. Identification of an ancient endogenous retrovirus, predating the divergence of the placental mammals. Philos Trans $R$ Soc Lond B Biol Sci (2013) 368:20120503. doi:10.1098/rstb.2012.0503 methodological heterogeneity, and the generally small study samples, including subjects with various diagnoses.

\section{CONCLUSION}

Human endogenous retroviruses provide an intriguing new avenue of research into the constituent factors involved in schizophrenia. Establishment of a possible causal relationship may lead to the development of new treatment strategies. Such strategies may involve elimination of triggering environmental factors or direct inhibition of HERV RNA or proteins.

\section{AUTHOR CONTRIBUTIONS}

Both authors have reviewed the literature, structured the information, developed conclusions, and wrote the manuscript.

\section{FUNDING}

This work was supported by the University of Bern.

14. Turner G, Barbulescu M, Su M, Jensen-Seaman MI, Kidd KK, Lenz J Insertional polymorphisms of full-length endogenous retroviruses in humans. Curr Biol (2001) 11:1531-5. doi:10.1016/S0960-9822(01)00455-9

15. Lander ES, Linton LM, Birren B, Nusbaum C, Zody MC, Baldwin J, et al. Initial sequencing and analysis of the human genome. Nature (2001) 409:860-921. doi: $10.1038 / 35057062$

16. Shin W, Lee J, Son SY, Ahn K, Kim HS, Han K. Human-specific HERV-K insertion causes genomic variations in the human genome. PLoS One (2013) 8:e60605. doi:10.1371/journal.pone.0060605

17. Marchi E, Kanapin A, Magiorkinis G, Belshaw R. Unfixed endogenous retroviral insertions in the human population. J Virol (2014) 88:9529-37. doi:10.1128/JVI.00919-14

18. Bannert N, Kurth R. The evolutionary dynamics of human endogenous retroviral families. Annu Rev Genomics Hum Genet (2006) 7:149-73. doi:10.1146/ annurev.genom.7.080505.115700

19. Blomberg J, Benachenhou F, Blikstad V, Sperber G, Mayer J. Classification and nomenclature of endogenous retroviral sequences (ERVs): problems and recommendations. Gene (2009) 448:115-23. doi:10.1016/j.gene.2009.06.007

20. Mayer J, Blomberg J, Seal RL. A revised nomenclature for transcribed human endogenous retroviral loci. Mob DNA (2011) 2:7. doi:10.1186/1759-8753-2-7

21. Jern P, Coffin JM. Effects of retroviruses on host genome function. Annu Rev Genet (2008) 42:709-32. doi:10.1146/annurev.genet.42.110807.091501

22. Cohen CJ, Lock WM, Mager DL. Endogenous retroviral LTRs as promoters for human genes: a critical assessment. Gene (2009) 448:105-14. doi:10.1016/j. gene.2009.06.020

23. Kapusta A, Kronenberg Z, Lynch VJ, Zhuo X, Ramsay L, Bourque G, et al. Transposable elements are major contributors to the origin, diversification, and regulation of vertebrate long noncoding RNAs. PLoS Genet (2013) 9:e1003470. doi:10.1371/journal.pgen.1003470

24. Forsman A, Yun Z, Hu L, Uzhameckis D, Jern P, Blomberg J. Development of broadly targeted human endogenous gammaretroviral pol-based real time PCRs Quantitation of RNA expression in human tissues. J Virol Methods (2005) 129:16-30. doi:10.1016/j.jviromet.2005.04.016

25. Goke J, Lu X, Chan YS, Ng HH, Ly LH, Sachs F, et al. Dynamic transcription of distinct classes of endogenous retroviral elements marks specific populations of early human embryonic cells. Cell Stem Cell (2015) 16:135-41. doi:10.1016/j. stem.2015.01.005

26. Lavialle C, Cornelis G, Dupressoir A, Esnault C, Heidmann O, Vernochet $\mathrm{C}$, et al. Paleovirology of 'syncytins', retroviral env genes exapted for a role in placentation. Philos Trans R Soc Lond B Biol Sci (2013) 368:20120507. doi:10.1098/rstb.2012.0507

27. Perron H, Lazarini F, Ruprecht K, Pechoux-Longin C, Seilhean D, Sazdovitch $\mathrm{V}$, et al. Human endogenous retrovirus (HERV)-W ENV and GAG 
proteins: physiological expression in human brain and pathophysiological modulation in multiple sclerosis lesions. J Neurovirol (2005) 11:23-33. doi:10.1080/13550280590901741

28. Weis S, Llenos IC, Sabunciyan S, Dulay JR, Isler L, Yolken R, et al. Reduced expression of human endogenous retrovirus (HERV)-W GAG protein in the cingulate gyrus and hippocampus in schizophrenia, bipolar disorder, and depression. J Neural Transm (2007) 114:645-55. doi:10.1007/ s00702-006-0599-y

29. Fei C, Atterby C, Edqvist PH, Ponten F, Zhang WW, Larsson E, et al. Detection of the human endogenous retrovirus ERV3-encoded Env-protein in human tissues using antibody-based proteomics. J R Soc Med (2014) 107:22-9. doi:10.1177/0141076813509981

30. Kassiotis G. Endogenous retroviruses and the development of cancer. J Immunol (2014) 192:1343-9. doi:10.4049/jimmunol.1302972

31. Christensen T. Association of human endogenous retroviruses with multiple sclerosis and possible interactions with herpes viruses. Rev Med Virol (2005) 15:179-211. doi:10.1002/rmv.465

32. Nelson P, Rylance P, Roden D, Trela M, Tugnet N. Viruses as potential pathogenic agents in systemic lupus erythematosus. Lupus (2014) 23:596-605. doi:10.1177/0961203314531637

33. Volkman HE, Stetson DB. The enemy within: endogenous retroelements and autoimmune disease. Nat Immunol (2014) 15:415-22. doi:10.1038/ni.2872

34. Deb-Rinker P, Klempan TA, O'Reilly RL, Torrey EF, Singh SM. Molecular characterization of a MSRV-like sequence identified by RDA from monozygotic twin pairs discordant for schizophrenia. Genomics (1999) 61:133-44. doi:10.1006/geno.1999.5946

35. Deb-Rinker P, O'Reilly RL, Torrey EF, Singh SM. Molecular characterization of a $2.7-\mathrm{kb}, 12 \mathrm{q} 13$-specific, retroviral-related sequence isolated by RDA from monozygotic twin pairs discordant for schizophrenia. Genome (2002) 45:381-90. doi:10.1139/g01-152

36. Perron H, Hamdani N, Faucard R, Lajnef M, Jamain S, Daban-Huard C, et al. Molecular characteristics of Human Endogenous Retrovirus type-W in schizophrenia and bipolar disorder. Transl Psychiatry (2012) 2:e201. doi:10.1038/tp.2012.125

37. Yolken RH, Karlsson H, Yee F, Johnston-Wilson NL, Torrey EF. Endogenous retroviruses and schizophrenia. Brain Res Brain Res Rev (2000) 31:193-9. doi:10.1016/S0165-0173(99)00037-5

38. Otowa T, Tochigi M, Rogers M, Umekage T, Kato N, Sasaki T. Insertional polymorphism of endogenous retrovirus HERV-K115 in schizophrenia. Neurosci Lett (2006) 408:226-9. doi:10.1016/j.neulet.2006.09.004

39. Dickerson F, Rubalcaba E, Viscidi R, Yang S, Stallings C, Sullens A, et al. Polymorphisms in human endogenous retrovirus K-18 and risk of type 2 diabetes in individuals with schizophrenia. Schizophr Res (2008) 104:121-6. doi:10.1016/j.schres.2008.05.005

40. Nyegaard M, Demontis D, Thestrup BB, Hedemand A, Sorensen KM, Hansen T, et al. No association of polymorphisms in human endogenous retrovirus K18 and CD48 with schizophrenia. Psychiatr Genet (2012) 22:146-8. doi:10.1097/YPG.0b013e328353953c

41. Karlsson H, Bachmann S, Schroder J, McArthur J, Torrey EF, Yolken RH. Retroviral RNA identified in the cerebrospinal fluids and brains of individuals with schizophrenia. Proc Natl Acad Sci U S A (2001) 98:4634-9. doi:10.1073/ pnas.061021998

42. Frank O, Giehl M, Zheng C, Hehlmann R, Leib-Mosch C, Seifarth W. Human endogenous retrovirus expression profiles in samples from brains of patients with schizophrenia and bipolar disorders. J Virol (2005) 79:10890-901. doi:10.1128/JVI.79.17.10890-10901.2005

43. Diem O, Schaffner M, Seifarth W, Leib-Mosch C. Influence of antipsychotic drugs on human endogenous retrovirus (HERV) transcription in brain cells. PLoS One (2012) 7:e30054. doi:10.1371/journal.pone.0030054

44. Karlsson H, Schroder J, Bachmann S, Bottmer C, Yolken RH. HERV-W-related RNA detected in plasma from individuals with recent-onset schizophrenia or schizoaffective disorder. Mol Psychiatry (2004) 9:12-3. doi:10.1038/ sj.mp. 4001439

45. Huang WJ, Liu ZC, Wei W, Wang GH, Wu JG, Zhu F. Human endogenous retroviral pol RNA and protein detected and identified in the blood of individuals with schizophrenia. Schizophr Res (2006) 83:193-9. doi:10.1016/j. schres.2006.01.007

46. Yao Y, Schroder J, Nellaker C, Bottmer C, Bachmann S, Yolken RH, et al. Elevated levels of human endogenous retrovirus-W transcripts in blood cells from patients with first episode schizophrenia. Genes Brain Behav (2008) 7:103-12. doi:10.1111/j.1601-183X.2007.00334.x

47. Huang W, Li S, Hu Y, Yu H, Luo F, Zhang Q, et al. Implication of the env gene of the human endogenous retrovirus $\mathrm{W}$ family in the expression of BDNF and DRD3 and development of recent-onset schizophrenia. Schizophr Bull (2011) 37:988-1000. doi:10.1093/schbul/sbp166

48. Weis S, Llenos IC, Dulay JR, Verma N, Sabunciyan S, Yolken RH. Changes in region- and cell type-specific expression patterns of neutral amino acid transporter 1 (ASCT-1) in the anterior cingulate cortex and hippocampus in schizophrenia, bipolar disorder and major depression. J Neural Transm (Vienna) (2007) 114:261-71. doi:10.1007/s00702-006-0544-0

49. Perron H, Mekaoui L, Bernard C, Veas F, Stefas I, Leboyer M. Endogenous retrovirus type W GAG and envelope protein antigenemia in serum of schizophrenic patients. Biol Psychiatry (2008) 64:1019-23. doi:10.1016/j. biopsych.2008.06.028

50. Hart DJ, Heath RG, Sautter FJ Jr, Schwartz BD, Garry RF, Choi B, et al. Antiretroviral antibodies: implications for schizophrenia, schizophrenia spectrum disorders, and bipolar disorder. Biol Psychiatry (1999) 45:704-14. doi:10.1016/S0006-3223(98)00229-7

51. Lillehoj EP, Ford GM, Bachmann S, Schroder J, Torrey EF, Yolken RH. Serum antibodies reactive with non-human primate retroviruses identified in acute onset schizophrenia. J Neurovirol (2000) 6:492-7. doi:10.3109/13550280009091949

52. Dickerson F, Lillehoj E, Stallings C, Wiley M, Origoni A, Vaughan C, et al. Antibodies to retroviruses in recent onset psychosis and multi-episode schizophrenia. Schizophr Res (2012) 138:198-205. doi:10.1016/j. schres.2012.03.037

53. Hurst T, Magiorkinis G. Activation of the innate immune response by endogenous retroviruses. J Gen Virol (2015) 96(Pt 6):1207-18. doi:10.1099/ jgv.0.000017

54. Zeng M, Hu Z, Shi X, Li X, Zhan X, Li XD, et al. MAVS, cGAS, and endogenous retroviruses in T-independent B cell responses. Science (2014) 346:1486-92. doi:10.1126/science.346.6216.1486

55. Rolland A, Jouvin-Marche E, Viret C, Faure M, Perron H, Marche PN. The envelope protein of a human endogenous retrovirus- $\mathrm{W}$ family activates innate immunity through CD14/TLR4 and promotes Th1-like responses. J Immunol (2006) 176:7636-44. doi:10.4049/jimmunol.176.12.7636

56. Perron H, Jouvin-Marche E, Michel M, Ounanian-Paraz A, Camelo S, Dumon A, et al. Multiple sclerosis retrovirus particles and recombinant envelope trigger an abnormal immune response in vitro, by inducing polyclonal Vbeta16 T-lymphocyte activation. Virology (2001) 287:321-32. doi:10.1006/ viro.2001.1045

57. Antony JM, van Marle G, Opii W, Butterfield DA, Mallet F, Yong VW, et al. Human endogenous retrovirus glycoprotein-mediated induction of redox reactants causes oligodendrocyte death and demyelination. Nat Neurosci (2004) 7:1088-95. doi:10.1038/nn1319

58. Miller BJ, Culpepper N, Rapaport MH. C-reactive protein levels in schizophrenia: a review and meta-analysis. Clin Schizophr Relat Psychoses (2014) 7:223-30. doi:10.3371/CSRP.MICU.020813

59. Lavillette D, Marin M, Ruggieri A, Mallet F, Cosset FL, Kabat D. The envelope glycoprotein of human endogenous retrovirus type $\mathrm{W}$ uses a divergent family of amino acid transporters/cell surface receptors. J Virol (2002) 76:6442-52. doi:10.1128/JVI.76.13.6442-6452.2002

60. Rasko JE, Battini JL, Gottschalk RJ, Mazo I, Miller AD. The RD114/simian type D retrovirus receptor is a neutral amino acid transporter. Proc Natl Acad Sci U S A (1999) 96:2129-34. doi:10.1073/pnas.96.5.2129

61. Angelucci F, Brene S, Mathe AA. BDNF in schizophrenia, depression and corresponding animal models. Mol Psychiatry (2005) 10:345-52. doi:10.1038/ sj.mp. 4001637

62. Li S, Liu ZC, Yin SJ, Chen YT, Yu HL, Zeng J, et al. Human endogenous retrovirus $\mathrm{W}$ family envelope gene activates the small conductance $\mathrm{Ca} 2+$-activated $\mathrm{K}+$ channel in human neuroblastoma cells through CREB. Neuroscience (2013) 247:164-74. doi:10.1016/j.neuroscience.2013.05.033

63. Perron H, Bertrand J-B, Faucard R, Jouanou J, Medina J, Firouzi R, et al. Neurobehavioral abnormalities induced by HERV-W Env in mouse brain: an animal model for HERV-W pathogenicity in schizophrenia? Neurol Psychiatry Brain Res (2014) 20:19-20. doi:10.1016/j.npbr.2014.01.163

64. HegyiH.GABBR1 hasa HERV-WLTRinits regulatory region-a possibleimplication for schizophrenia. Biol Direct (2013) 8:5. doi:10.1186/1745-6150-8-5 
65. Suntsova M, Gogvadze EV, Salozhin S, Gaifullin N, Eroshkin F, Dmitriev $\mathrm{SE}$, et al. Human-specific endogenous retroviral insert serves as an enhancer for the schizophrenia-linked gene PRODH. Proc Natl Acad Sci U S A (2013) 110:19472-7. doi:10.1073/pnas.1318172110

66. Kelley D, Rinn J. Transposable elements reveal a stem cell-specific class of long noncoding RNAs. Genome Biol (2012) 13:R107. doi:10.1186/ gb-2012-13-11-r107

67. Qureshi IA, Mehler MF. Emerging roles of non-coding RNAs in brain evolution, development, plasticity and disease. Nat Rev Neurosci (2012) 13:528-41. doi:10.1038/nrn3234

68. Barry G. Integrating the roles of long and small non-coding RNA in brain function and disease. Mol Psychiatry (2014) 19:410-6. doi:10.1038/mp.2013.196

69. Liao Q, Wang Y, Cheng J, Dai D, Zhou X, Zhang Y, et al. DNA methylation patterns of protein coding genes and long noncoding RNAs in female schizophrenic patients. Eur J Med Genet (2015) 58:95-104. doi:10.1016/j. ejmg.2014.12.001

70. Ren Y, Cui Y, Li X, Wang B, Na L, Shi J, et al. A co-expression network analysis reveals lncRNA abnormalities in peripheral blood in early-onset schizophrenia. Prog Neuropsychopharmacol Biol Psychiatry (2015) 63:1-5. doi:10.1016/j. pnpbp.2015.05.002

71. Pavlicek A, Paces J, Elleder D, Hejnar J. Processed pseudogenes of human endogenous retroviruses generated by LINEs: their integration, stability, and distribution. Genome Res (2002) 12:391-9. doi:10.1101/gr.216902

72. Bundo M, Toyoshima M, Okada Y, Akamatsu W, Ueda J, Nemoto-Miyauchi T, et al. Increased 11 retrotransposition in the neuronal genome in schizophrenia. Neuron (2014) 81:306-13. doi:10.1016/j.neuron.2013.10.053

73. Johnston JB, Silva C, Holden J, Warren KG, Clark AW, Power C. Monocyte activation and differentiation augment human endogenous retrovirus expression: implications for inflammatory brain diseases. Ann Neurol (2001) 50:434-42. doi:10.1002/ana.1131

74. Young GR, Mavrommatis B, Kassiotis G. Microarray analysis reveals global modulation of endogenous retroelement transcription by microbes. Retrovirology (2014) 11:59. doi:10.1186/1742-4690-11-59

75. Frank O, Jones-Brando L, Leib-Mosch C, Yolken R, Seifarth W. Altered transcriptional activity of human endogenous retroviruses in neuroepithelial cells after infection with Toxoplasma gondii. J Infect Dis (2006) 194:1447-9. doi:10.1086/508496
76. Nellaker C, Yao Y, Jones-Brando L, Mallet F, Yolken RH, Karlsson H Transactivation of elements in the human endogenous retrovirus $\mathrm{W}$ family by viral infection. Retrovirology (2006) 3:44. doi:10.1186/1742-4690-3-44

77. Li F, Nellaker C, Sabunciyan S, Yolken RH, Jones-Brando L, Johansson AS, et al. Transcriptional derepression of the ERVWE1 locus following influenza A virus infection. J Virol (2014) 88:4328-37. doi:10.1128/JVI.03628-13

78. Cardno AG, Owen MJ. Genetic relationships between schizophrenia, bipolar disorder, and schizoaffective disorder. Schizophr Bull (2014) 40:504-15. doi:10.1093/schbul/sbu016

79. De Peri L, Crescini A, Deste G, Fusar-Poli P, Sacchetti E, Vita A. Brain structural abnormalities at the onset of schizophrenia and bipolar disorder: a meta-analysis of controlled magnetic resonance imaging studies. Curr Pharm Des (2012) 18:486-94. doi:10.2174/138161212799316253

80. Perron H, Lang A. The human endogenous retrovirus link between genes and environment in multiple sclerosis and in multifactorial diseases associating neuroinflammation. Clin Rev Allergy Immunol (2010) 39:51-61. doi:10.1007/ s12016-009-8170-x

81. Antony JM, Deslauriers AM, Bhat RK, Ellestad KK, Power C. Human endogenous retroviruses and multiple sclerosis: innocent bystanders or disease determinants? Biochim Biophys Acta (2011) 1812:162-76. doi:10.1016/j. bbadis.2010.07.016

82. Garcia-Montojo M, Dominguez-Mozo M, Arias-Leal A, Garcia-Martinez A, De las Heras V, Casanova I, et al. The DNA copy number of human endogenous retrovirus-W (MSRV-type) is increased in multiple sclerosis patients and is influenced by gender and disease severity. PLoS One (2013) 8:e53623. doi:10.1371/journal.pone.0053623

Conflict of Interest Statement: The authors declare that the research was conducted in the absence of any commercial or financial relationships that could be construed as a potential conflict of interest.

Copyright (C) 2016 Slokar and Hasler. This is an open-access article distributed under the terms of the Creative Commons Attribution License (CC BY). The use, distribution or reproduction in other forums is permitted, provided the original author(s) or licensor are credited and that the original publication in this journal is cited, in accordance with accepted academic practice. No use, distribution or reproduction is permitted which does not comply with these terms. 